Pacific Journal of Mathematic 


\title{
A NOTE ON COMPACT SEMIRINGS WHICH ARE MULTIPLICATIVE SEMILATTICES
}

\author{
P. H. Karvellas
}

\begin{abstract}
The topic of this note is the structure of a topological semiring in which a semilattice (commutative, idempotent and associative) multiplication, with identity and connected upper sets, has been postulated. Assuming the topology to be compact, additions compatible with the multiplication can be characterized for certain canonical subsets of the semiring. In particular instances the characterization of addition can be extended to the entire semiring itself.
\end{abstract}

Certain subintervals, arising naturally from the analysis when the underlying space is the interval $[0,1]$, are generalized to continuum subsemirings of an arbitrary semiring possessing a semilattice multiplication with identity. The addition in the minimal additive ideal can be specified precisely and each additive subgroup is a single element. If the minimal additive ideal and the set of additive idempotents coincide, a complete description of the semiring addition is possible in terms of homomorphisms of the multiplicative semigroup. The same procedure can be employed when the space is an interval on the real line.

A topological semiring $(S,+, \cdot)$ is a Hausdorff space $S$ on which are defined topological semigroups $(S,+)$ and $(S, \cdot)$, for addition and multiplication, such that $x(y+z)=x y+x z$ and $(x+y) z=x z+y z$ for all $x, y$, and $z$ in $S$. This structure will be investigated under the restrictions that $(S, \cdot)$ is a topological semilattice, with identity 1 and multiplicative zero element 0 , the set $S$ is compact and upper sets $M(x)=\{y: x y=x\}$ are connected for each $x$ in $S$. Such a semiring will be called a semilattice semiring or SL-semiring. Multiplication is therefore commutative and idempotent in a semilattice semiring and an induced partial order, with closed graph, results from defining $x \leqq y$ if $x=x y$.

Unless specifically altered, both $(S,+, \cdot)$ and $S$ shall refer to semilattice semirings in the analysis which follows.

Particular examples of $S L$-semirings appear in [5], where $S$ is the real number interval $[0,1]$. The characterization of such interval $S L$-semirings is given in Example 1 and employs two continuous functions satisfying certain required conditions on subsets of $[0,1]$. A more general space and analysis will, of course, be subject to rather more exaggerated ambiguities.

Ideals will be semigroup ideals in the sense of [1] and kernels 
(minimal ideals) with be written as $K[+]$ and $K[\cdot]$. In the compact case kernels are nonvoid and closed [7], as are the idempotent sets $E[+]=\{x: x=x+x\}$ and $E[\cdot]=\left\{x: x=x^{2}\right\}$. The union of all additive subgroups will be written as $H[+]$ and for $t$ in $E[+]$ the maximal additive subgroup with identity element $t$ is $H[+](t)$. For a positive integer $n$ and element $x, n x$ denotes the $n$-fold sum of $x$. Equivalently $n x$ is the product of two elements of the semiring. The element $(1+1)$ will be written as $p$.

For an element $x$ let $L(x)=\{y: x y=y\}$ and $M(x)=\{y: x y=x\}$. If $x \leqq y$, that is if $x=x y$, then define $C(x, y)=\{z: x \leqq z \leqq y\}=$ $M(x) \cap L(y)=y \cdot M(x)$. In any $S L$-semiring, $M(x)$ is connected, implying the connectivity of $C(x, y)$ for $x \leqq y$. It is trivial to verify that $C(x, y)$ is a subsemiring if and only if $x \in E[+]$. Lastly, from $S=E[\cdot], x+y=(x+y)^{2}=x+p(x y)+y$ for all $x, y \in S$.

2. Connected subsemirings of a semilattice semiring. In Example 1 is given the characterization, obtained in [5], of all $S L$ semirings on the interval $[0,1]$. The resulting subintervals $[0, e]$, $[e, f],[f, p]$, and $[p, 1]$ have obvious generalizations to an arbitrary $S L$-semiring defined on a general topological space.

EXAMPLE 1. Let $S=[0,1]$ with multiplication $x y=\min (x, y)$. Any compatible semiring addition, with $x+y=y$ in $K[+]$, can be characterized as follows. Pick arbitrary elements $e, f$, and $p$ in $[0,1]$, where $0 \leqq e \leqq f \leqq p \leqq 1$. Let $F:[0, p] \rightarrow[e, 1]$ and $G:[0, p] \rightarrow[f, 1]$ be continuous functions such that

(1) $F$ is the identity on $[e, p]$;

(2) $F$ decreases on $[0, e]$ and $G$ decreases on $[0, f]$;

(3) for $x \in[0, p], p G(x)=\max (f, p F(x))$.

The addition on $S$ is defined by

$$
\begin{aligned}
x+y & =p & & x, y \geqq p \\
& =x F(y) & & y \leqq x, y<p \\
& =y G(x) & & x<y, x<p .
\end{aligned}
$$

The subintervals $[0, e],[e, f],[f, p]$, and $[p, 1]$ are connected subsemirings with the additions below.

$$
\begin{array}{llll}
x+y=\max (x, y) & x, y \in[0, e] & s+k=k & k \in[e, f], s \in S \\
x+y=x y & x, y \in[f, p] & x+y=p & x, y \in[p, 1] .
\end{array}
$$

The additive kernel $K[+]$ is the subinterval $[e, f]$, while $E[+]=[0, p]$.

In any $S L$-semiring $(S, \cdot)$ is commutative and the kernel $K[\cdot]$ must reduce to a singleton, denoted hereafter by 0 [4]. It is easy to verify that $2 x=4 x$ for each $x$ in $S$ and from [3] both $E[+]$ and 
$H[+]$ are multiplicative ideals, requiring $0 \in E[+]$. Because $(S, \cdot)$ has an identity, $E[+]$ is closed under addition [3], and both $E[+]$ and $H[+]$ are connected [8]. Alternatively $p=1+1=p^{2}=p+p$ and $p x=x+x$ for each $x$ in $S$. The map $x \rightarrow p x$ is continuous and $M(0)=S$ is connected. Hence $E[+]=p S$ is connected. As will be proven subsequently, $E[+]=H[+]$. Noting that $S=M(0)$ and is connected, we have the result below.

THEOREM 1. Let $(S,+, \cdot)$ be a semilattice semiring.

(1) $K[\cdot]=\{0\} \subseteq E[+]$ and $S$ is a connected set.

(2) $E[+]=\{x+x: x \in S\}$ and is an additive subsemigroup.

(3) $E[+]$ and $H[+]$ are connected multiplicative ideals.

The next result characterizes the operations in the minimal additive ideal $K[+]$.

TheOREM 2. Let $(S,+, \cdot)$ be a semilattice semiring. Then:

(1) $K[+]$ is a subsemiring of $S$ contained in $E[+]$.

(2) There exist elements $e$ and $f$ in $S$ such that $K[+]=C(e, f)$ and $f=1+k+1$ for each element $k \in K[+]$.

(3) $K[+]=(S+e)+(e+S)$, with each element $z$ in $K[+]$ uniquely of the form $z_{1}+z_{2}$, where $z_{1} \in S+e$ and $z_{2} \in e+S$. Moreover, for elements $x_{1}, x_{2}$ in $S+e$ and $y_{1}, y_{2}$ in $e+S$, the kernel operations are given by

$$
\begin{aligned}
\left(x_{1}+y_{1}\right)+\left(x_{2}+y_{2}\right) & =x_{1}+y_{2} \\
\left(x_{1}+y_{1}\right) \cdot\left(x_{2}+y_{2}\right) & =x_{1} x_{2}+y_{1} y_{2} \\
(e+S) \cap(S+e) & =\{e\} .
\end{aligned}
$$

Proof. Because $S^{2} \cap K[+]$ is nonvoid, the additive kernel is a subsemiring using a result from [6]. From $S=E[\cdot]$ and Theorem 1 of [7] each additive subgroup is totally disconnected. However, $K[+]$ is the union of the connected maximal subgroups $H[+](t)=$ $t+S+t$ for $t$ in $K[+] \cap E[+][8]$ : hence $H[+](t)=\{t\}$ for each $t \in K[+] \cap E[+]$ and thus $K[+] \subseteq E[+]$. The compact, commutative subsemigroup $(K[+], \cdot)$ has a multiplicative kernel which is a single point. Let $\{e\}$ denote this kernel. Then $f=1+e+1$ is in $K[+]$ and, for each element $k$ in $K[+], e \leqq k$ while

$$
\begin{gathered}
f k=k+e+k \in k+S+k=H[+](k)=\{k\} \\
1+k+1=f(1+k+1)=f+k+f \in H[+](f)=\{f\}
\end{gathered}
$$

proving that $1+K[+]+1=\{f\}$ and $K[+] \leqq C(e, f)$. For any element $x \in C(e, f), \quad x=x f=x(1+e+1)=x+e+x \in C(e, f) \cap K[+]$ 
and hence $K[+]=C(e, f)$. The characterization of addition in $K[+]$ follows directly from Theorem 1.3.10 of [4] and the triviality of maximal additive subgroups in $K[+]$. For $x_{1}, x_{2}$ in $S+e$ and $y_{1}, y_{2}$ in $e+S$ we have that $x_{1}=x_{1}+e, y_{1}=e+y_{1}$ and $H[+](e)=e+$ $S+e=\{e\}$, implying therefore that $(e+S) \cap(S+e) \subseteq H[+](e)$ and that

$$
\begin{aligned}
\left(x_{1}+y_{1}\right) \cdot\left(x_{2}+y_{2}\right) & =x_{1} x_{2}+y_{1} x_{2}+x_{1} y_{2}+y_{1} y_{2} \\
& =x_{1} x_{2}+\left(e+y_{1}\right) x_{2}+\left(x_{1}+e\right) y_{2}+y_{1} y_{2} \\
& =x_{1} x_{2}+e+y_{1} x_{2}+x_{1} y_{2}+e+y_{1} y_{2} \\
& =x_{1} x_{2}+e+y_{1} y_{2} \\
& =x_{1} x_{2}+y_{1} y_{2} .
\end{aligned}
$$

The subsets of interest are the following: $E[+]=p S, K[+]=$ $C(e, f), M(p)=\{x: p x=p\}, L(e)=e S$ and $1+S+1$. Both $E[+]$ and $K[+]$ have been shown to be connected subsemirings from the preceding arguments. As proven in Theorem 4, the requirement that $M(x)$ be connected for each $x$ in $S$ results in $p=p+1$ and implies triviality of addition in $M(p)$. If the restriction on upper sets is removed, partial results can still be obtained.

THEOREm 3. Let $(T,+, \cdot)$ be a compact semiring, with $E[+]=$ $\{q\}$, such that $(T, \cdot)$ is a semilattice with identity 1. Then:

(1) $1+x=x+1$ and $q=1+1=x+q+x$ for all $x$ in $T$.

(2) $(T,+)$ is commutative.

(3) $T+T$ is the additive kernel.

Proof. Since $T=E[\cdot], 1+1=(1+1)^{2}=(1+1)+(1+1) \in E[+]$ and thus $q=1+1$. Moreover, $K[\cdot] \leqq E[+]=\{q\}$. Hence $q=q x$ for each $x$ in $T$. It is easily shown that $1+x=(1+x)^{2}=1+3 x=$ $q+1+x$ for each element $x$ of $T$. Analogously $x+1=x+1+q$. As a result one obtains the equations

$$
\begin{aligned}
(x+1) \cdot(1+x) & =x(1+x)+(1+x)=q+1+x=1+x \\
& =(x+1)+(x+1) x=x+1+q=x+1 .
\end{aligned}
$$

Moreover, $x+q+x=x+q x+x=x(2 q)=q$. In a similar manner it can be proven that $x+y=(x+y) \cdot(y+x)=y+x$ for all $x$ and $y$ in $T$. Addition in $T$ is therefore commutative.

Lastly, because $(T,+)$ is a compact semigroup with a single idempotent element, $K[+]=H[+](q)=T+q+T$ [8]. Thus, for $x$ and $y$ in $T, x+y=(x+y)^{2}=x+q(x y)+y=x+q+y \in K[+]$. Therefore $T+T \subseteq T+q+T=K[+]$, implying that $K[+]=T+T$.

THEOREM 4. Let $(S,+, \cdot)$ be a semilattice semiring, $p=1+1$. Then: 
(1) $(M(p),+, \cdot)$ is a subsemiring with trivial addition.

(2) $L(e)=e S$ is a distributive topological lattice.

Proof. From $M(p)=\{x: p x=p\}$ it is clear that $M(p)$ is a continuum subsemiring with a single additive idempotent. Theorem 3 applies and it is now only necessary to note that the additive kernel of the subsemiring $M(p)$ is the connected additive group $M(p)+p+$ $M(p)$. However, $M(p) \subseteq E[\cdot]$ and from [7] this group must also be totally disconnected. Consequently $M(p)+M(p)=\{p\}$ and $p=1+$ $1=p+1=1+p$.

Recall that $K[+]=C(e, f)$ where $\{e\}$ is the multiplicative kernel of the subsemiring $K[+]$. The subcontinuum $e S=L(e)$ is a subsemiring with identity $e$ and $e=e+x=x+e$ for each $x=e x$ in $e S$. Thus for elements $x$ and $y$ of $e S$ we obtain

$$
\begin{aligned}
& (x+y) x=x e+x y=x(e+y)=x e=x \\
& (x+y) y=x y+e y=(x+e) y=e y=y .
\end{aligned}
$$

Therefore, $x+y \in M(x) \cap M(y)$ and for any $t \in M(x) \cap M(y)$ it follows that $t(x+y)=t x+t y=x+y$. That is, $x+y$ is the least upper bound of $x$ and $y$ in the partial order defined by the semilattice multiplication and consequently $(e S,+, \cdot)$ is a lattice. Since multiplication distributes over addition, both lattice distributive laws hold.

Corollary 5. Let $(S,+, \cdot)$ be a semilattice semiring. If $E[+]=\{0\}$ then $S+S=\{0\}$.

THEOREM 6. Let $(S,+, \cdot)$ be a semilattice semiring and let $f$ denote the maximal element of the additive kernel, while $p=1+1$. Then:

(1) These are equivalent statements.

(a) $(E[+],+)$ is commutative.

(b) $x+p=p+x$ for all $x$ in $E[+]$.

(c) $x+p=p+x$ for all $x$ in $S$.

(2) If $(E[+],+)$ is commutative, then $(E[+],+, \cdot)$ is a topological lattice if and only if $f=p$.

Proof. Recall that $E[+]$ is a connected subsemiring. For any $x$ in $S$ we have that $x+p=(x+p)^{2}=(p+1) x+p$ and $(p+1) x \epsilon$ $E[+]$. Thus if $x+p=p+x$ for $x$ in $E[+]$, the same result holds in $S$, and vice versa.

Clearly, (a) $\rightarrow($ b). Assume that elements of $S$ commute with $p$ under addition. For $x, y \in E[+], x=x+x=p x, x y=p x y, y=y+$ $y=p y$ and thus the equations below are obtained. 


$$
\begin{gathered}
x+y=(x+y)^{2}=x+x y+y=x(p+y)+y=x y+(x+y) \\
=x+(x+p) y=(x+y)+x y \\
y+x=(y+x)^{2}=y+x y+x=x y+(y+x) \\
=(y+x)+x y .
\end{gathered}
$$

It follows that

$$
\begin{aligned}
(x+y) \cdot(y+x) & =x(y+x)+y(y+x)=x y+(x+y)+x y \\
& =(x+y) y+(x+y) x=x y+(y+x)+x y
\end{aligned}
$$

which implies that $(E[+],+)$ is commutative.

Assume now that addition in $E[+]$ is commutative. Because distinct idempotents in $K[+]$ do not commute in the compact case [4], we obtain $K[+]=\{f\}$. If $f=p$ then, from Theorem $4, E[+]$ is a distributive topological lattice. Conversely, if $E[+]$ is a lattice then, since one distributive law holds, $E[+]$ is a distributive lattice. Therefore, because $a=a(a+b)=a+(a b)$ for all $a$ and $b$ in the lattice $E[+]$, we obtain

$$
p=p+p f=p^{2}+p f=p(p+f)=p f=f .
$$

The following example illustrates the general idempotent semilattice semiring with commutative addition which can be constructed on an interval.

ExAmple 2. Let $S=[z, p]$ be an interval of real numbers with min multiplication. Fix an element $f$ in $S$ and denote the subintervals $[z, f]$ by $A$ and $[f, p]$ by $B$ respectively. If $\{f\}$ is the additive kernel of an idempotent and commutative addition semiring on $[z, p]$, then $B=p+B$ and $x+y=\min (x, y)$ in $B$, while $x+y=\max (x, y)$ in $A$. The map $f: S \rightarrow B$ defined by $f(x)=1+x$ is continuous and is the identity on $B$. Moreover, $f$ reverses order on $A(x y=x$ in $A$ implies $f(x) \cdot f(y)=f(y)$ in $B)$. Any such addition on $S$ is therefore given by the characterization

$$
\begin{aligned}
x+y & =x F(y) & & y \leqq x \\
& =y F(x) & & x<y
\end{aligned}
$$

where $F: S \rightarrow B$ is continuous, the identity on $B$ and order-reversing on $A$.

The existence of the three elements $p(=1+1)$, $e$ and $f$, where $K[+]=C(e, f)$, has allowed the characterization of addition in $M(p)$, $K[+]$ and $L(e)$. The next result completes the description of connected subsemirings which are analogues of the subintervals appearing in Example 1. 
THEOREM 7. Let $(S,+, \cdot)$ be a semilattice semiring, $p=1+1$ and $K[+]=C(e, f)$ for elements $e \leqq f$ in $E[+]$. Then:

(1) $H[+]=E[+]$ and each additive subgroup is a single point.

(2) $1+S+1=1+E[+]+1 \subseteq M(f) \cap E[+]$ with addition given by $x+y=x y=y+x$.

(3) For $x \in 1+S+1, y \in M(p), x+y=x=y+x$.

(4) $M(f)+K[+]+M(f)=\{f\}$.

(5) $e+1 \geqq e+s$ and $1+e \geqq s+e$ for all $s$ in $S$.

(6) $S+p+S \subseteq E[+]$.

(7) The boundary $B$ of $E[+]$ is connected.

Proof. For $t \in E[+]$ the maximal additive subgroup $H[+](t)$ is a subsemiring since $t=t^{2}[2]$. Moreover, $H[+](t) \cong M(t)$ since for each $x \in H[+](t), \quad t x \in E[+] \cap H[+](t)=\{t\}$. Consequently $x+x=$ $p x=t$ and therefore $x=x+t=(1+p) x=t$ for each $x$ in $H[+](t)$. Hence $H[+] \subseteq E[+]$ and each additive subgroup is a single element.

Clearly $1+E[+] \subseteq 1+S$ and, because $1+x=(1+x)^{2}=1+p x$ for each element $x$, the reverse inclusion also holds. Similarly $S+$ $1=E[+]+1$ and for each element $x$ of $S$ we have that

$$
\begin{aligned}
1+x+1=(1+x+1)^{2} & =(1+x+1)+3 x+(1+x+1) \\
& =p(1+x)+p(x+1) \\
& =p(1+x+1) \in E[+] .
\end{aligned}
$$

In addition it follows that $f=f+x+f=f(1+x+1)$, implying that $1+S+1 \leqq M(f) \cap E[+]$. For any two elements $x$ and $y$ of $1+$ $S+1, p x=p x+1$ and $p y=1+p y$ and hence

$$
\begin{aligned}
x+y=(x+y)^{2}=x+p(x y)+y & =x(1+p y)+y \\
& =p(x y)+y=p(x y)=x y
\end{aligned}
$$

and in a similar manner $y+x=x y$. Moreover, for $x \in 1+S+1$ and $y \in M(p)$ we obtain

$$
x+y=x y+y=(x+1) y=x y=x .
$$

For elements $k \in K[+]$, and $m, n \in M(f)$, we have that

$$
\begin{gathered}
k+n=f(k+n)=k+f n=k+f \\
m+k+n=(f+k)+n=f+k+f=f .
\end{gathered}
$$

Consequently $M(f)+K[+]+M(f)=\{f\}$.

For any element $s \in S$ it follows that $(e+s) \leqq(e+1)$ since

$$
(e+1)(e+s)=e+e s+e+s=e+s
$$

and similarly $(s+e) \leqq(1+e)$. In addition, for elements $x$ and $y$ of 
$S, p x+1=x+1,1+y=1+p y$ and therefore $x+p+y=p(x+$ $1+y) \in E[+]$, implying $S+p+S \subseteq E[+]$.

Lastly, consider the set $T=S \backslash E[+]$, which is connected since for each $t$ in $T$ the interval $C(t, 1) \subseteq T$. Consequently $p T$ is also connected and $p T \subseteq E[+]$. For $x$ in $T$ let $R(x)=\{y: p x=p y\}$. Then $R(x) \cap E[+]=\{p x\}, x \in R(x)$ and it is easily verified that $R(x)$ is a compact subsemiring of $S$. Moreover, $C(p x, y) \subseteq R(x)$ for each $y$ in $R(x)$, implying that $R(x)$ is connected. Suppose now that $p x$ is contained in the interior of $E[+]$. There then exists an open set $U$, containing $p x$, and contained in $E[+]$. However, $U \cap R(x)=\{p x\}$ is an open and closed subset of the connected set $R(x)$. Consequently $p T$ is contained in the boundary $B$ of $E[+]$. It is now only necessary to note that if $r \in B$, then for any open set $W$ containing $r$ there exists an open set $V$, containing $r$, such that $p V \subseteq W$. Thus, since $V \cap T$ is nonempty, $r$ is a limit point of the connected set $p T$ and $B$ is connected.

Identification of the various connected subsemirings of a general semilattice semiring with the subintervals obtained in Example 1 yields the correspondences: $L(e)$ with $[0, e] ; M(p)$ with $[p, 1]$; and, $1+S+1$ with $[f, p]$. The addition in the additive kernel $K[+]$ of a general $S L$-semiring is that of a rectangular band [1], while the existence of a cutpoint in the Example 1 case produces either a left- or righttrivial addition [4].

The construction of "characterizing functions", as given in Example 1 , is apparently futile for a general semilattice semiring. However, as demonstrated below, the situation $K[+]=E[+]$ is amenable to this approach.

3. Semilattice semirings with $K[+]=E[+]$. In the case of $S L$-semiring with $K[+]=E[+]$ it is possible to obtain a complete characterization of the addition in terms of semilattice homomorphisms on the multiplicative semigroup. The following lemma establishes some preliminary results. Then:

Lemma 8. Let $S$ be a semilattice semiring with $K[+]=E[+]$.

(1) $S+S \subseteq E[+]$.

(2) For $x, y \in S, \quad\{x+y\}=x+S+y, 0+x \leqq 0+1 \leqq x+1$ and $x+0 \leqq 1+0 \leqq 1+x$.

(3) For $k \in K[+], k+M(f)=\{k+1\},\{f\}=M(f)+k+M(f)$.

(4) The maps $x \stackrel{F}{\longrightarrow}(1+x)$ and $x \stackrel{G}{\longrightarrow}(x+1)$ are semiring homomorphisms with $F(x+y)=F(y)$ and $G(x+y)=G(x)$. Addition in $S$ is given by 


$$
x+y=G(x) \cdot F(y) .
$$

(5) For $x, y \in S, \quad M(x+0) \cap M(0+x)=M(p x)$ and $M(f)=$ $M(1+x) \cap M(y+1)=M(x+1) \cap M(1+y)$.

Proof. Noting that $p=f$ and that $E[+](=K[+])$ is both an additive and multiplicative ideal, we have the result

$$
x+y=(x+y)^{2}=x+p(x y)+y \in K[+]
$$

for each $x$ and $y$ in $S$. Recall that $H[+](p x)=x+S+x=\{p x\}$ and therefore, using both distributive laws, we obtain

$$
\begin{aligned}
(x+1) \cdot(x+0+1) & =(x+x)+0+(x+1)=x+1 \\
& =p x+(x+0+1)=x+0+1 .
\end{aligned}
$$

Analogously $1+x=1+0+x$. Using $\{p(x y)\}=x y+S+x y$ the following equations hold.

$$
\begin{aligned}
x+f+y & =p(x+f+y)=p(x+1+y)=x+1+y \\
& =(x+f x+x y)+(x f+f+y f)+(x y+f y+y) \\
& =f x+f(x y)+f y \\
& =x+p(x y)+y=x+y .
\end{aligned}
$$

Therefore, for any $x$ and $y$ in $S$, it follows that

$$
\begin{aligned}
x+S+y=f(x+S+y) & =(x+S)+(S+y) \\
& =(x+1+S)+(S+1+y) \\
& =x+f+y=x+y .
\end{aligned}
$$

For each $x$ in $S, 0+x=x(0+1) \leqq 0+1$. Similarly we have that $(x+1) \cdot(0+1)=0+x+0+1=0+1 \leqq x+1$. For $k$ in $K[+]$ and $m$ in $M(f)(=M(p)), k+m=p(k+m)=k+1$. Analogously $M(f)+k=\{1+k\}$, thereby establishing (3) as a special case of Theorem 7 (4).

Consider the maps $F, G: S \rightarrow K[+]$ defined by $F(x)=1+x, G(x)=$ $x+1$. Both are semiring homomorphisms and addition in $S$ is given by

$$
x+y=x+1+x y+y=(x+1) \cdot(1+y)=G(x) \cdot F(y) .
$$

Lastly, $x+0,0+x \leqq p x$. And, if $t \in M(x+0) \cap M(0+x)$, then $t x+0=x+0,0+x=0+t x$, implying the result

$$
t(p x)=t x+0+t x=x+0+x \in x+S+x=\{p x\} .
$$

Similarly, $M(1+x) \cap M(y+1)=M(x+1) \cap M(1+y)=M(f)$.

The next example describes a general semilattice semiring under 
the restriction that the additive kernel $K[+]$ is the set $E[+]$ of additive idempotents.

EXAMPLE 3 . Let $(S, \cdot)$ be a compact topological semilattice, with identity element 1 and connected upper sets. Let $p$ be any fixed element of $S$. If $F$ and $G$ are continuous semilattice homomorphisms from $S$ into $p S$ such that

(a) $(F \circ F)(x)=F(x),(G \circ G)(x)=G(x)$ for all $x$ in $S$;

(b) $F(x) G(x)=p x$ for all $x$ in $S$;

(c) $(F \circ G)(x)=(G \circ F)(x)=p$ for all $x$ in $S$ :

(where "०" denotes composition) and an addition is defined on $S$ by

$$
x+y=G(x) F(y)
$$

for all $x$ and $y$ in $S$, then $(S,+, \cdot)$ is a semilattice semiring with additive kernel $K[+]=E[+]=p S$.

THEOREM 9. Let $(S, \cdot)$ be a compact topological semilattice, with identity element 1 and connected upper sets.

(a) For any fixed element $p$ of $S$, and homomorphisms $F$ and $G$ into $p S$ defining an addition $(+)$ as in Example $3,(S,+, \cdot)$ is a semilattice semiring with $K[+]=E[+]=p S$.

(b) Conversely, if $(+)$ is the addition of a semilattice semiring on the set $S$, with $K[+]=E[+]$ and addition compatible with the given semilattice multiplication, then the maps $F, G: S \rightarrow E[+]$ defined by $F(x)=1+x, G(x)=x+1$ satisfy the properties of Example 3 when $p$ is taken to be the element $(1+1)$ of $S$.

Proof. The verification of part (a) is trivial, albeit tedious. If, on the other hand, $(S,+, \cdot)$ is a semilattice semiring with $E[+]=$ $K[+]$, and the maps $F$ and $G$ are as defined, then both are continuous multiplicative homomorphisms, as proven in Lemma 8. Clearly $F(F(x))=1+F(x)=p+x=1+x=F(x)$ and $G(G(x))=G(x)$ for all $x$ in $S$. Analogously $F(x) \cdot G(x)=(1+x) \cdot(x+1)=x+1+x=$ px. Moreover, $(F \circ G)(x)=1+G(x)=1+x+1=p$. Lastly, as shown in Lemma 8, addition satisfies the definition given in Example 3.

The final two results, presented without proof, describe a $S L$ semiring in which $E[+]=K[+]$ and $S \backslash E[+] \subseteq M(1+0) \cup M(0+1)$. Note that the latter condition is not sufficient to describe the characterization on the interval given in Example 1.

LEMMA 10. Let $S$ be a semilattice semiring with $E[+]=K[+]$. Then these are equivalent statements for an element $x$ of $S$.

(1) $1+x=f[x+1=f]$ :

(2) $x \in M(0+1)[x \in M(1+0)]$ : 
(3) $\quad p x=x+1[p x=1+x]$.

THEOREM 11. Let $(S,+, \cdot)$ be a semilattice semiring, with $E[+]=K[+]$, in which $S \backslash E[+] \subseteq M(1+0) \cup M(0+1)$. Then addition in $S$ is given by:

$$
\begin{aligned}
x+y & =p y & & x, y \in M(1+0) \\
& =p x & & x, y \in M(0+1) \\
& =f & & x \in M(1+0), y \in M(0+1) \\
& =p(x y) & & x \in M(0+1), y \in M(1+0) \\
& =G(x) \cdot y & & x \in E[+], y \in M(1+0) \\
& =F(y) & & x \in M(1+0), y \in E[+] \\
& =G(x) & & x \in E[+], y \in M(0+1) \\
& =x \cdot F(y) & & x \in M(0+1), y \in E[+]
\end{aligned}
$$

where $F, G: S \rightarrow E[+]$ are defined by $F(x)=1+x, G(x)=x+1$.

The author would like to express his appreciation to Professor Michael Friedberg for his suggestions and criticism.

\section{REFERENCES}

1. A. H. Clifford and G. B. Preston, The Algebraic Theory of Semigroups, Volumes I and II, The American Mathematical Society, Mathematical Surveys No. 7, 1961.

2. M. O. Poinsignon Grillet, Subdivision rings of a semiring, Fund. Math., 67 (1970), 67-74.

3. P. H. Karvellas, Inversive semirings, J. Australian Math. Soc., (to appear).

4. A. B. Paalman de Miranda, Topological Semigroups, Mathematisch Centrum, Amsterdam, 1964.

5. K. R. Pearson, Certain topological semirings in $R_{1}$, J. Australian Math. Soc., 8 (1968), 171-182.

6. - The three kernels of a compact semiring, J. Australian Math. Soc., 10 (1969), 299-319.

7. J. Selden, A note on compact semirings, Proc. Amer. Math. Soc., 15 (1964), 882-886.

8. A. D. Wallace, The structure of topological semigroups, Bull. Amer. Math. Soc., 61 (1955), 95-122.

Received March 28, 1973 and in revised form July 24, 1973.

University of Alberta, CANada 



\section{PACIFIC JOURNAL OF MATHEMATICS}

EDITORS

RICHARD ARENS (Managing Editor)

University of California

Los Angeles, Calıfornia 90024

R. A. Beaumont

University of Washington

Seattle, Washington 98105
J. DugundJI

Department of Mathematics

University of Southern California

Los Angeles, California 90007

D. Gilbarg and J. Milgram

Stanford University

Stanford, California 94305

\section{ASSOCIATE EDITORS}
E. F. BECKENBACH
B. H. NeumanN
F. WOLF
K. YOSHIDA

\section{SUPPORTING INSTITUTIONS}

UNIVERSITY OF BRITISH COLUMBIA

CALIFORNIA INSTITUTE OF TECHNOLOGY

UNIVERSITY OF CALIFORNIA

MONTANA STATE UNIVERSITY

UNIVERSITY OF NEVADA

NEW MEXICO STATE UNIVERSITY

OREGON STATE UNIVERSITY

UNIVERSITY OF OREGON

OSAKA UNIVERSITY
UNIVERSITY OF SOUTHERN CALIFORNIA

STANFORD UNIVERSITY

UNIVERSITY OF TOKYO

UNIVERSITY OF UTAH

WASHINGTON STATE UNIVERSITY

UNIVERSITY OF WASHINGTON

AMERICAN MATHEMATICAL SOCIETY NAVAL WEAPONS CENTER 


\section{Pacific Journal of Mathematics}

\section{Vol. 55, No. $1 \quad$ September, 1974}

Robert Lee Anderson, Continuous spectra of a singular symmetric

differential operator on a Hilbert space of vector-valued functions . . . $\quad 1$

Michael James Cambern, The isometries of $L^{p}(X, K) \ldots \ldots \ldots \ldots \ldots . . \ldots$

R. H. Cameron and David Arne Storvick, Two related integrals over spaces of continuous functions ................................

Gary Theodore Chartrand and Albert David Polimeni, Ramsey theory and

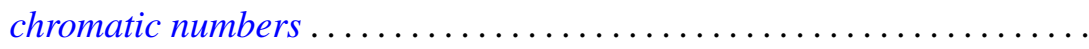

John Deryck De Pree and Harry Scott Klein, Characterization of collectively compact sets of linear operators ...................

John Deryck De Pree and Harry Scott Klein, Semi-groups and collectively compact sets of linear operators ....................... 55

George Epstein and Alfred Horn, Chain based lattices.............. 65

Paul Erdős and Ernst Gabor Straus, On the irrationality of certain series . . 85

Zdeněk Frolík, Measurable uniform spaces................... 93

Stephen Michael Gagola, Jr., Characters fully ramified over a normal

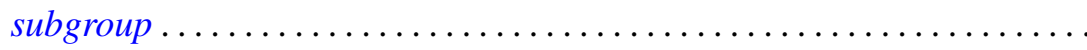

Frank Larkin Gilfeather, Operator valued roots of abelian analytic

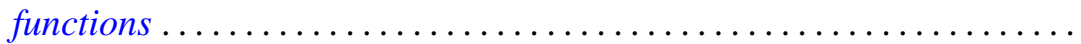

D. S. Goel, A. S. B. Holland, Cyril Nasim and B. N. Sahney, Best approximation by a saturation class of polynomial operators

James Secord Howland, Puiseux series for resonances at an embedded

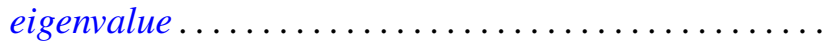

David Jacobson, Linear GCD equations .................

P. H. Karvellas, A note on compact semirings which are multiplicative

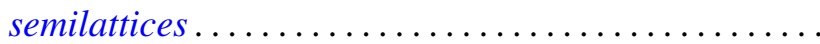

Allan Morton Krall, Stieltjes differential-boundary operators. II . .

D. G. Larman, On the inner aperture and intersections of convex sets

S. N. Mukhopadhyay, On the regularity of the $P^{n}$-integral and its application to summable trigonometric series ....... .

Dwight Webster Read, On $(J, M, m)$-extensions of Boolean algebras ....

David Francis Rearick, Multiplicativity-preserving arithmetic power series.

Indranand Sinha, Characteristic ideals in group algebras

Charles Thomas Tucker, II, Homomorphisms of Riesz spaces . . .

Kunio Yamagata, The exchange property and direct sums of indecomposable injective modules. 